\title{
Mild hypothermia attenuates post-resuscitation brain injury through a V-ATPase mechanism in a rat model of cardiac arrest
}

\author{
J.C. Zhang ${ }^{1,2 *}$, W. Lu ${ }^{4 *}$, X.M. Xie ${ }^{1}$, H. Pan $^{3}$, Z.Q. Wu ${ }^{1}$ and G.T. Yang \\ ${ }^{1}$ Department of Emergency, Tongji Hospital, Tongji Medical College, \\ Huazhong University of Science and Technology, Wuhan, Hubei, China \\ ${ }^{2}$ Department of Emergency, Xinhua Hospital, \\ Shanghai Jiaotong University School of Medicine, Shanghai, China \\ ${ }^{3}$ Department of Nephrology, Tongji Hospital, Tongji Medical College, \\ Huazhong University of Science and Technology, Wuhan, Hubei, China \\ ${ }^{4}$ Department of Otolaryngology, \\ Shanghai Jiao Tong University Affiliated Sixth People's Hospital, \\ Shanghai Jiao Tong University School of Medicine, Shanghai, China \\ *These authors contributed equally to this study. \\ Corresponding author: G.T. Yang \\ E-mail: guangtiany@hotmail.com \\ Genet. Mol. Res. 15 (2): gmr.15027729 \\ Received September 25, 2015 \\ Accepted December 14, 2015 \\ Published June 3, 2016 \\ DOI http://dx.doi.org/10.4238/gmr.15027729
}

\begin{abstract}
Although therapeutic hypothermia is an effective treatment for post-resuscitation brain injury after cardiac arrest (CA), the underlyingmechanism remains unclear. Vacuolar $\mathrm{H}^{+}$-ATPase(V-ATPase) plays a key role in cellular adaption to a hypoxic environment. This study sought to evaluate the effect of mild hypothermia on V-ATPase and its involvement in neuroprotection after CA. Male Sprague-Dawley rats were subjected to a 6-min CA, resuscitated successfully, and then assigned to either the normothermia (NT) group or the hypothermia (HT) group. Rats were further divided into 2 subgroups based on the time of euthanasia, either 3 or 24 h after CA (NT-3 h, HT-3 h; NT-24 h,
\end{abstract}


HT-24 h). Mild hypothermia was induced following CA and maintained at $33^{\circ} \mathrm{C}$ for $2 \mathrm{~h}$. Neurologic deficit scores were used to determine the status of neurological function. Brain specimens were analyzed by TUNEL assay, western blotting, and immunohistochemistry. V-ATPase activity was estimated by subtracting total ATP hydrolysis from the bafilomycin-sensitive activity. Mild hypothermia improved the neurological outcome (HT-24 h: $34.3 \pm 16.4$ vs NT-24 h: $50.3 \pm 17.4$ ) and significantly decreased neurocyte apoptosis $24 \mathrm{~h}$ after resuscitation. Mild hypothermia significantly increased V0a1 compared to NT-3 h; V0a1 expression was associated with a decrease in the cleaved caspase 3 expression. These findings suggested that mild hypothermia inhibits CA-induced apoptosis in the hippocampus, which may be associated with reduced V-ATPase impairment. These data provide new insights into the protective effects of hypothermia in vivo.

Key words: Cardiac arrest; Cardiopulmonary resuscitation; Electrical stimulation; Vacuolar $\mathrm{H}^{+}$-ATPase (V-ATPase); Mild hypothermia

\section{INTRODUCTION}

Cardiac arrest (CA) is a major public health issue and remains the leading cause of death in the United States (Stecker et al., 2014). Of those who successfully achieve a restoration of spontaneous circulation (ROSC), only 2 to $10 \%$ survive without major neurological impairments (Knapp et al., 2013). Currently, the only therapeutic option to improve neurological outcome after CA is mild hypothermia (Knapp et al., 2011). Although it is widely used after CA, the mechanisms underlying mild hyperthermia and its induction of ischemic tolerance are not fully understood.

Vacuolar $\mathrm{H}^{+}$-ATPase (V-ATPase) is a multimeric enzyme complex composed of V0, a membrane sector, which triggers proton translocation, and V1, a cytoplasmic sector, which hydrolyzes ATP (Poëa-Guyon et al., 2006). The V0 domain consists of at least 5 different subunits (a, c, c', c', and d), while the V1 domain contains up to 8 additional subunits (A-H) (Su et al., 2003). V-ATPase is an ATP-driven proton pump involved in the maintenance of intracellular $\mathrm{pH}$ homeostasis, anti-oxidative activity, and the $\mathrm{H}^{+}$gradient across the vacuolar membrane, which is crucial for neurotransmitter transportation and $\mathrm{Ca}^{2+}$ uptake from the cellular plasma (Nishi and Forgac, 2002; Chintagari et al., 2010). Recent studies have found that V-ATPase activity enhancement favored the survival of tumor cells in hypoxic and acidic environments (Marino et al., 2010; Chung et al., 2011; Fogarty et al., 2014). Therefore, in this study, we hypothesized that mild hypothermia may inhibit neuronal apoptosis by an upregulation of V-ATPase following CA.

V-ATPase is typically expressed in neurons, and changes in its expression and function have been reported in synaptic vesicle swelling and neurotransmission (Crider and Xie, 2003; Shin et al., 2010). V-ATPase dysfunction and defects in vesicular acidification have recently been recognized as important mechanisms in various human diseases, such as autoimmune disorders, cancer, and neurological disorders (Marshansky and Futai, 2008). These studies indicate that V-ATPase may play an essential role in brain injury after cardiac arrest. 


\section{MATERIAL AND METHODS}

The experimental protocol was approved by the Institutional Animal Care and Use Committee of Huazhong University of Science and Technology. All animals were treated humanely in accordance with the Guidance Suggestions for the Care and Use of Laboratory Animals published by Ministry of Science and Technology of the People's Republic of China.

\section{Animal preparation and cardiac arrest procedure}

Fifty-eight Sprague-Dawley male rats (body weight: 300-350 g; supplier: Animal Experimental Center of Wuhan University, Wuhan, China) were used in this study. Prior to use, the rats were fasted overnight but had free access to water. Rats were anesthetized with pentobarbital sodium $(45 \mathrm{mg} / \mathrm{kg}$, ip $)$. Rat tracheas were orally intubated and mechanically ventilated (HX-300S, Chengdu Taimeng Technology Corp., Chengdu, China). Two PE 50 catheters were inserted into the right femoral artery and vein. Arterial blood pressure, heart rate, and electrocardiograms were continuously recorded (RM6240BD, Chengdu Instrument Factory, Chengdu, China). A rectal temperature probe was inserted into the rats' rectums, and a heating lamp was used to maintain core temperatures $\left(37^{\circ} \pm 0.5^{\circ} \mathrm{C}\right)$.

Cardiac arrest was induced in this rat model by transcutaneous electrical epicardium, which was described previously (Lin et al., 2010). Briefly, 2 disposable acupuncture needles were inserted into the visceral pericardium between the fourth rib of the left sternal border and the third rib of the right sternal border. The needles were connected to a stimulator electrode (RM6240BD, Chengdu Instrument Factory). The electrical current (60 Hz and $2 \mathrm{~mA}$ ) lasted for $3 \mathrm{~min}$ to prevent spontaneous defibrillation. Cardiac arrest was defined as the loss of blood pressure $(\leq 20 \mathrm{mmHg})$, ventricular fibrillation, and asystole or pulseless electrical activity after the electrical stimulation stopped. After $6 \mathrm{~min}$ of CA, advanced cardiac life support, including ventilation $\left(100 \% \mathrm{FiO}_{2}, 100\right.$ breaths $\left./ \mathrm{min}, 0.65 \mathrm{~mL} / 100 \mathrm{~g}\right)$, chest compression (200 times/min), and adrenaline administration $(2 \mu \mathrm{g} / 100 \mathrm{~g})$, were started. Spontaneous circulation was confirmed by a sinus rhythm with a mean aortic pressure greater than $60 \mathrm{mmHg}$ for at least $10 \mathrm{~min}$. In successfully resuscitated rats, the ventilation rate was adjusted according to blood gas analyses. After spontaneous circulation was restored, mechanical ventilation and hemodynamic monitoring were continued with $21 \%$ oxygen for the next $3 \mathrm{~h}$. All catheters and wounds were removed or sutured after $3 \mathrm{~h}$.

\section{Experimental design}

Immediately after ROSC, animals were assigned to either the normothermic (NT, N = $20)$ or hypothermic (HT, $N=20)$ group by a random-sequence generator. Rats in these groups were further divided into 2 subgroups based on the time of euthanasia, either 3 or $24 \mathrm{~h}$ after CA (NT-3 h, N = 10; HT-3 h, N = 10; NT-24 h, N = 10; HT-24 h, N = 10). Animals in the sham group $(\mathrm{N}=10)$ received the same procedures as the experimental rats with the exception of $\mathrm{CA}$ and hypothermia. Data were obtained and analyzed only from animals that survived to the pre-defined endpoints.

The body temperature of the NT group was maintained at $37^{\circ} \pm 0.5^{\circ} \mathrm{C}$ throughout the experiment using a heating lamp. Mild hypothermia was induced in the HT group with a cool alcohol gauze and an electric fan $\left(33^{\circ}-34^{\circ} \mathrm{C}\right)$ beginning $30 \mathrm{~min}$ after resuscitation 
and maintained for $2 \mathrm{~h}$ (Park et al., 2013). After cooling, the rats were gradually warmed to normothermia at a rate of $0.5^{\circ}-1^{\circ} \mathrm{C} / \mathrm{h}$ by stopping the fan and starting the heating lamp. The temperature of animals receiving hypothermia typically rose to $37^{\circ} \mathrm{C}$ by the 6th to 8 th $\mathrm{h}$ following ROSC. All experimental procedures are summarized in Figure 1A.

\section{Neurological deficit score (NDS)}

To evaluate the neurological state of successfully resuscitated animals, the NDS was assessed $24 \mathrm{~h}$ after cardiopulmonary resuscitation (CPR). The NDS was performed as described previously (Katz et al., 1995). Briefly, the NDS consisted of 5 components: general behavioral function (consciousness and respiration, 40 points), cranial nerve function (20 points), motor function (10 points), sensory function (10 points), and coordination (including balance beam walk, 20 points). The scoring system ranged from 0 (normal) to 100 (brain death). The investigator was blinded to the 2 groups.

\section{TUNEL staining}

TUNEL staining was performed according to the manufacturer instructions (Roche, Germany) in order to identify apoptotic cells in the paraffin-embedded brain sections. Five high-power fields (400X) were randomly selected, and the number of apoptotic cells was counted for each field.

\section{Immunofluorescence}

V-ATPase expression was examined in the sham, NT-3 h, and HT- $3 \mathrm{~h}$ groups. The rats were deeply anesthetized and transcardially perfused with ice-cold normal saline followed by freshly prepared $4 \%$ paraformaldehyde. The heads were dissected, and the brains were removed and immersed in $4 \%$ paraformaldehyde overnight. After immersion in 20\% sucrose solution at $4^{\circ} \mathrm{C}$ overnight, the brain was embedded in optimum cutting temperature compound (Sakura Finetek, Torrance, CA, USA) and frozen at $-20^{\circ} \mathrm{C}$. A cryostat microtome (Leica, Wetzlar, Germany) was used to cut $50-\mu \mathrm{m}$ sections. After washing in phosphate-buffered saline, the sections were incubated with $10 \%$ normal goat serum and $0.1 \%$ Triton-X 100 for 30 min at room temperature. After incubation with the primary antibody (rabbit anti-V-ATPase V0a1 antibody, 1:200, Santa Cruz Biotechnology, Dallas, TX, USA; mouse anti-cleaved caspase 3 antibody, 1:500; Proteintech, Chicago, IL, USA) overnight at $4^{\circ} \mathrm{C}$, the sections were washed and incubated for $1 \mathrm{~h}$ at room temperature with secondary anti-rabbit Alexa Fluor 568 (1:400, Molecular Probes; Invitrogen, Waltham, MA, USA) and anti-mouse Alexa Fluor 488 (1:400, Molecular Probes; Invitrogen). The slides were counterstained with DAPI (1:800, Invitrogen) for $5 \mathrm{~min}$ and washed. The slides were then analyzed using a confocal microscope (Olympus FV1000, Japan).

\section{Western blot analysis}

A 70-mg frozen hippocampal sample was homogenized in RIPA lysis buffer (Beyotime, Nanjing, China); the extracted proteins were quantified using a BCA reagent (Beyotime). A total of $30 \mu \mathrm{g}$ protein for each sample was run on $8 \%$ gels by SDS-PAGE and 
transferred to polyvinylidene fluoride membranes (Millipore-Upstate, Billerica, MA, USA). The membranes were blocked in 5\% non-fat milk for $2 \mathrm{~h}$ at room temperature followed by incubation with a primary antibody (V-ATPase V0a1: 1:200, Santa Cruz Biotechnology; cleaved caspase 3: 1:600, Proteintech Group; $\beta$-actin: 1:2000, Proteintech Group) at $4{ }^{\circ} \mathrm{C}$ overnight. After washing with TBS-T (1X TBS, $0.05 \%$ Tween-20), the membranes were incubated with secondary antibody (1:5000, Proteintech Group) for $1 \mathrm{~h}$ at $37^{\circ} \mathrm{C}$. The protein band signals were imaged and analyzed with Image-Pro Plus (Version 6.0). The quantitative data are reported as the target protein $\mathrm{OD} / \beta$-actin $\mathrm{OD}$ ratio.

\section{V-ATPase enzymatic activity}

V-ATPase activity was estimated by subtracting the total ATP hydrolysis from the bafilomycin $\mathrm{A}_{1}$-sensitive activity, as previously described (Sennoune et al., 2004), with some modifications. Briefly, the total ATPase activities of the cortex and hippocampus were detected using an ATPase activity assay kit (MAK113, Sigma, St. Louis, MO, USA) according to the manufacturer directions. Frozen hippocampus samples $(50 \mathrm{mg})$ were homogenized in $1 \mathrm{~mL}$ ice-cold TBS buffer. The homogenate was centrifuged at 14,000 $g$ for $10 \mathrm{~min}$ at $4^{\circ} \mathrm{C}$. The clear supernatant was transferred to a clean tube and the protein concentrations were measured using BCA reagent (Beyotime). The reaction mix contained assay buffer $(20 \mu \mathrm{L})$, enzyme sample $(10 \mu \mathrm{L})$, and $4 \mathrm{mM} \mathrm{ATP}(10 \mu \mathrm{L})$, which was incubated for $30 \mathrm{~min}$ at $37^{\circ} \mathrm{C}$. The bafilomycin $\mathrm{A}_{1}$ assay reaction mix contained assay buffer $(20$ $\mu \mathrm{L})$, enzyme sample $(5 \mu \mathrm{L})$, and $50 \mathrm{nM}$ bafilomycin $\mathrm{A}_{1}(5 \mu \mathrm{L})$, which was incubated for $1 \mathrm{~h}$ at $37^{\circ} \mathrm{C}$; $4 \mathrm{mM}$ ATP $(10 \mu \mathrm{L})$ was added for $30 \mathrm{~min}$ at $37^{\circ} \mathrm{C}$ to determine the enzyme activity. The reagent $(200 \mu \mathrm{L})$ was added to each well, and the samples were incubated for an additional $30 \mathrm{~min}$ at $37^{\circ} \mathrm{C}$ to generate the colorimetric product. The absorbance intensity was measured using a microplate reader at $620 \mathrm{~nm}$. The V-ATPase activity is reported as $\mu \mathrm{mol} \mathrm{P}_{\mathrm{i}} \cdot \mu \mathrm{g}$ protein ${ }^{-1} \cdot \mathrm{min}^{-1}$.

\section{Statistical analysis}

Statistical analysis was performed with SPSS Statistics 17.0. All data, except for the survival rate, are reported as means \pm standard deviation (SD) and were analyzed with oneway analysis of variance (ANOVA), followed by post hoc Student-Newman-Keuls tests. The difference in the survival rate was analyzed by the Fisher exact test. A P $<0.05$ was considered statistically significant.

\section{RESULTS}

\section{Cardiac arrest, cardiopulmonary resuscitation, and survival}

The mean body temperatures of each group during the experiment are shown in Figure 1B. Before the induction of CA, there were no significant differences between the NT and HT groups in the arterial blood gas or hemodynamic variables. Cardiac arrest resulted in marked biochemical and physiologic disturbances. These alterations gradually improved after ROSC to the weaning of mechanic ventilation, although there were no significant differences between the NT and HT groups (Table 1). 


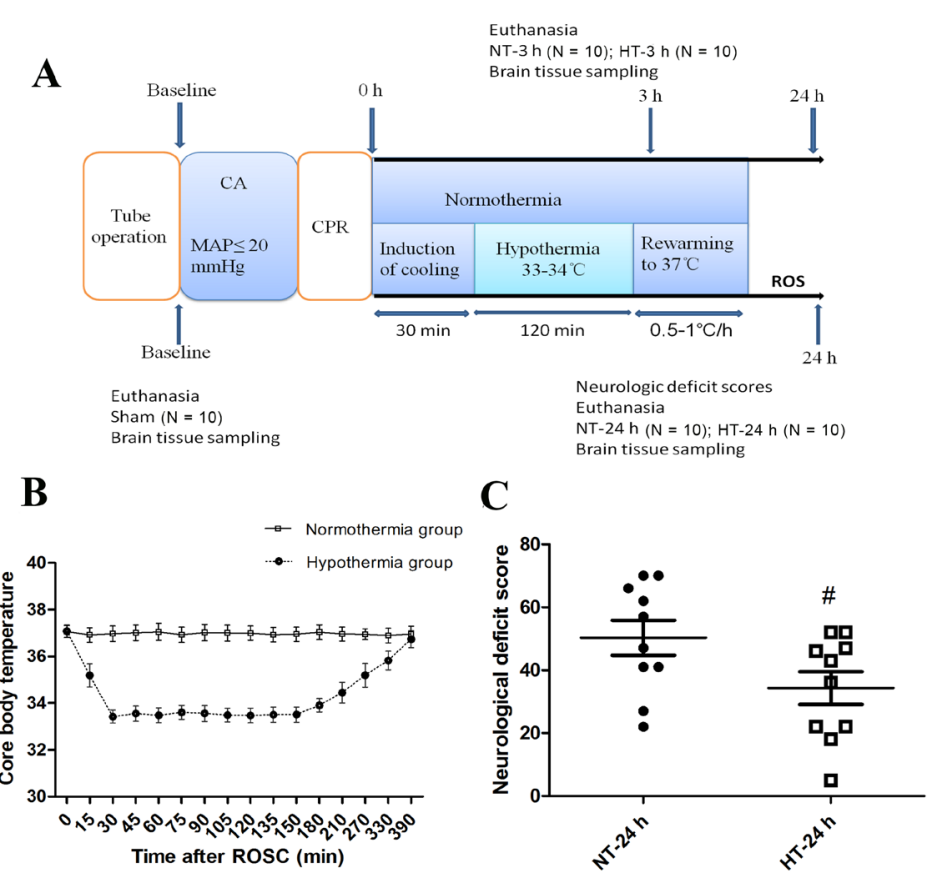

Figure 1. A. Procedure of experimental cardiac arrest $(\mathrm{CA})$ in animals. B. Time course of body temperature after cardiopulmonary resuscitation (CPR). Data are reported as means \pm SE. C. Neurological deficit score in normothermia (NT) and hypothermia (HT) groups at $24 \mathrm{~h}$ after resuscitation ( ${ }^{\#} \mathrm{P}<0.05$ vs NT- $24 \mathrm{~h}$ group).

Table 1. Physiological variables at baseline, $10 \mathrm{~min}$ after ROSC (return of spontaneous circulation) and $10 \mathrm{~min}$ after weaning of mechanical ventilation.

\begin{tabular}{|c|c|c|c|}
\hline & NT $(\mathrm{N}=20)$ & HT $(\mathrm{N}=20)$ & $P$ value \\
\hline \multicolumn{4}{|l|}{ Before cardiac arrest } \\
\hline Body weight $(\mathrm{g})$ & $328.7 \pm 15.9$ & $328.1 \pm 17.0$ & 0.90 \\
\hline $\mathrm{pH}$ & $7.42 \pm 0.02$ & $7.41 \pm 0.02$ & 0.36 \\
\hline $\mathrm{PaCO}_{2}(\mathrm{mmHg})$ & $35.2 \pm 2.0$ & $34.7 \pm 2.2$ & 0.46 \\
\hline $\mathrm{PaO}_{2}(\mathrm{mmHg})$ & $96.6 \pm 2.4$ & $96.9 \pm 2.1$ & 0.67 \\
\hline Lactate $(\mathrm{mM})$ & $0.7 \pm 0.14$ & $0.7 \pm 0.13$ & 0.82 \\
\hline Base excess & $-2.7 \pm 0.39$ & $-2.7 \pm 0.46$ & 0.74 \\
\hline Mean arterial pressure $(\mathrm{mmHg})$ & $104.3 \pm 7.2$ & $101.6 \pm 6.5$ & 0.22 \\
\hline Heart rate (bpm) & $375.2 \pm 16.6$ & $378.8 \pm 16.4$ & 0.50 \\
\hline \multicolumn{4}{|l|}{$10 \mathrm{~min}$ after ROSC } \\
\hline $\mathrm{pH}$ & $7.27 \pm 0.04$ & $7.27 \pm 0.03$ & 0.43 \\
\hline $\mathrm{PaCO}_{2}(\mathrm{mmHg})$ & $41.7 \pm 3.2$ & $42.1 \pm 4.5$ & 0.72 \\
\hline $\mathrm{PaO}_{2}(\mathrm{mmHg})$ & $316.2 \pm 14.2$ & $313.6 \pm 11.5$ & 0.53 \\
\hline Lactate $(\mathrm{mM})$ & $7.4 \pm 0.47$ & $7.2 \pm 0.45$ & 0.29 \\
\hline Base excess & $-13.0 \pm 2.7$ & $-13.7 \pm 3.2$ & 0.43 \\
\hline Mean arterial pressure $(\mathrm{mmHg})$ & $79.6 \pm 5.8$ & $78.2 \pm 5.0$ & 0.44 \\
\hline Heart rate $(\mathrm{bpm})$ & $306.2 \pm 16.1$ & $301.6 \pm 14.3$ & 0.35 \\
\hline \multicolumn{4}{|c|}{10 min after weaning of mechanical ventilation } \\
\hline $\mathrm{pH}$ & $7.37 \pm 0.04$ & $7.38 \pm 0.04$ & 0.38 \\
\hline $\mathrm{PaCO}_{2}(\mathrm{mmHg})$ & $37.7 \pm 4.3$ & $38.6 \pm 4.5$ & 0.52 \\
\hline $\mathrm{PaO}_{2}(\mathrm{mmHg})$ & $297.9 \pm 22.1$ & $294.5 \pm 17.2$ & 0.59 \\
\hline Lactate $(\mathrm{mM})$ & $1.5 \pm 0.2$ & $1.6 \pm 0.3$ & 0.32 \\
\hline Base excess & $3.2 \pm 0.58$ & $3.0 \pm 0.61$ & 0.24 \\
\hline Mean arterial pressure $(\mathrm{mmHg})$ & $98.4 \pm 9.4$ & $100.4 \pm 7.7$ & 0.47 \\
\hline Heart rate (bpm) & $349.2 \pm 11.3$ & $346.0 \pm 8.7$ & 0.31 \\
\hline
\end{tabular}

$\mathrm{NT}=$ normothermia; $\mathrm{HT}=$ hypothermia. 
After ROSC, the adequate spontaneous respiration gradually recovered over the course of $2 \mathrm{~h}$ in all rats. After catheter removal, most animals remained anesthetized, but were able to breath spontaneously. In the 3-h study, 2 rats in the NT-3-h group and 1 rat in the HT-3 $\mathrm{h}$ group died after ROSC before reaching the 3-h survival time point (NT-3 h: 10/12 (survived/ total number), HT-3 h: 10/11; $\mathrm{P}=0.59$ ). In the $24 \mathrm{~h}$ study, 2 rats in the NT-24 h group and 3 rats in the HT-24 h group died after ROSC before reaching 24-h survival (NT-24 h: 10/12, HT-24-h: 10/13, P = 0.68).

\section{Mild hypothermia improved neurological outcome $24 \mathrm{~h}$ after CPR}

Before the induction of CA, all rats had an NDS of 0 . The NDS was significantly improved in the HT-24 h group compared to the NT-24 h group $(34.3 \pm 16.4$ vs $50.3 \pm 17.4$, P $<0.05$; Figure 1C).

\section{Mild hypothermia prevented neurocyte apoptosis in the hippocampal CA1 region $24 \mathrm{~h}$ after ROSC}

There were significantly fewer TUNEL-positive cells in the HT-24 h group compared to the NT-24 h group (NT-24 h: $186 \pm 25.2$, HT-24 h: $141 \pm 20.9 ;$ P < 0.05; Figure 2).
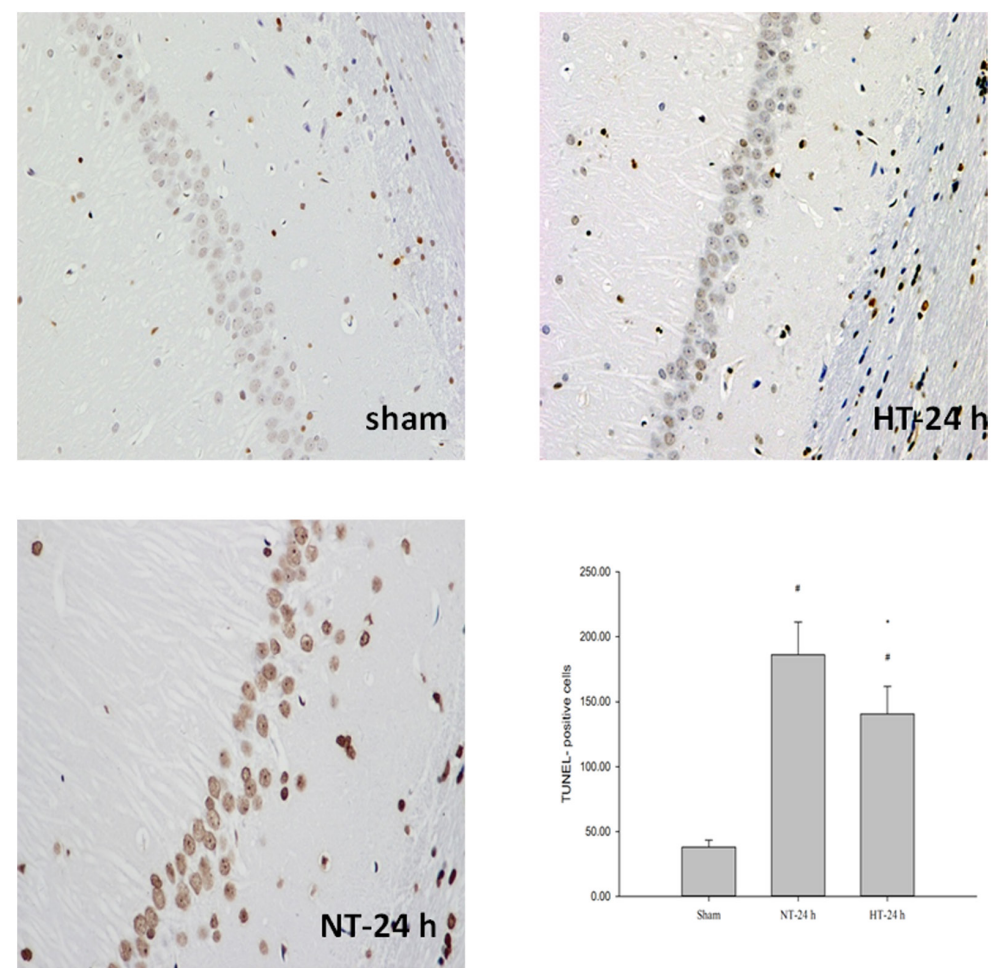

Figure 2. Representative images of hippocampal CA-1 sector by TUNEL staining in sham, NT-24 h, and HT-24 h groups. The number of TUNEL-positive cells was significantly less in the HT-24 $\mathrm{h}$ group than in the NT-24 $\mathrm{h}$ group (compared with sham, ${ }^{\#} \mathrm{P}<0.05$; compared with NT-24 h, ${ }^{*} \mathrm{P}<0.05$ ). 


\section{Mild hypothermia decreased the up-regulation of cleaved caspase 3 and restored the V0a1 subunit in the hippocampus $3 \mathrm{~h}$ after $\mathrm{CA}$}

After CA and ROSC, there was an increase in cleaved caspase 3 protein levels and a decrease in V0a1 subunit levels in the NT resuscitated rats (Figure 3A). Mild hypothermia significantly reduced the up-regulation of cleaved caspase 3 (NT-3 h: $0.49 \pm 0.02$, HT-3 h: $0.29 \pm$ $0.02 ; \mathrm{P}<0.05$ ) and restored V0a1 expression (NT-3 h: $0.36 \pm 0.05$, HT-3 h: $0.64 \pm 0.03 ; \mathrm{P}<0.05$ ) in the hippocampus $3 \mathrm{~h}$ after ROSC. These changes in the V0a1 subunit and activated caspase 3 protein levels were confirmed by immunohistochemical staining (Figure 3C). All proteins were located in the cytoplasm of neurocytes. The V0a1 immunoreactivity was notably higher in the HT-3 h group compared to the V0a1 immunoreactivity in the NT-3 h group.

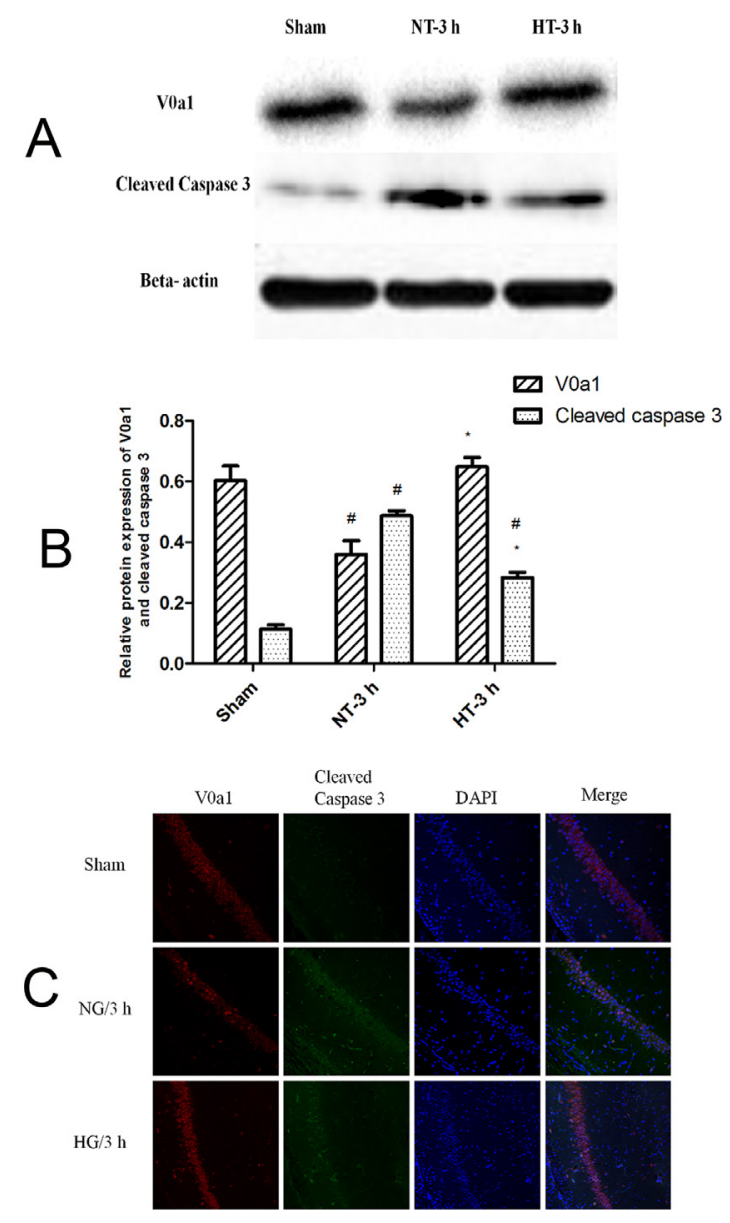

Figure 3. Mild hypothermia treatment after CA is associated with restored V0a1 subunit levels and decreased caspase 3 expression in the hippocampus $3 \mathrm{~h}$ after ROSC. A. Representative western blots of V0a1 subunit and cleaved caspase 3 protein levels. B. Mild hypothermia inhibited caspase 3 activation and restored V0a1 expression. C. Sections of brain were stained for V0a1 (red) and caspase 3 (green). Confocal microscopy was used to image CA-1 at 400X magnification (C). Compared with sham, ${ }^{\#} \mathrm{P}<0.05$; compared with NT-3 h, ${ }^{*} \mathrm{P}<0.05$. 


\section{Enzymatic activity}

V-ATPase activity (expressed as $\mu \mathrm{mol} \mathrm{P}_{\mathrm{i}} \cdot \mu \mathrm{g}$ protein ${ }^{-1} \cdot \mathrm{min}^{-1}$ ) in the hippocampus was $3.14 \pm 0.17$ (sham group, $\mathrm{N}=3$ ), $2.49 \pm 0.14(\mathrm{NT}-3 \mathrm{~h}$ group, $\mathrm{N}=3$ ) , $2.84 \pm 0.16$ (HT-3 h group, $\mathrm{N}=3), 3.31 \pm 0.27(\mathrm{NT}-24 \mathrm{~h}$ group, $\mathrm{N}=3)$ and $3.13 \pm 0.20(\mathrm{HT}-24 \mathrm{~h}$ group, $\mathrm{N}=3)$ (Figure 4). In the NT group, there was a down-regulation of V-ATPase activity $3 \mathrm{~h}$ after CPR $(\mathrm{P}<0.05$, compared to the sham group), which returned to baseline levels at $24 \mathrm{~h}$. These data indicate that mild hypothermia restored V-ATPase activity $3 \mathrm{~h}$ after ROSC $(\mathrm{P}<0.05$ compared to the NT-3 h group).

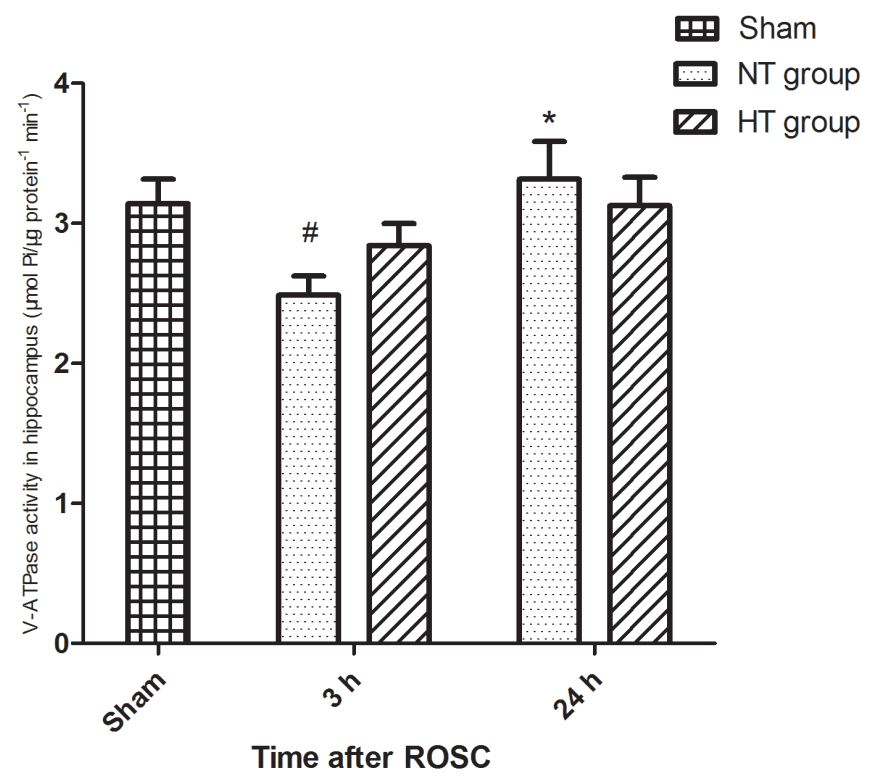

Figure 4. Statistical results for V-ATPase activity in sham 3 and $24 \mathrm{~h}$ after ROSC in the hippocampus (compared with the sham group, ${ }^{\mathrm{P}}<0.05$; compared with the NT-3 h group, $\left.{ }^{*} \mathrm{P}<0.05\right)$.

\section{DISCUSSION}

These data demonstrate that the treatment of whole body mild hypothermia 1) improves neurological outcome, 2) attenuates neuronal apoptosis in the hippocampus, even at $24 \mathrm{~h}$ following ROSC, 3) reduces cleaved caspase 3 levels, 4) up-regulates the expression of the V0a1 subunit, and 5) increases V-ATPase activity compared to the NT group $3 \mathrm{~h}$ after CA.

Previous animal and clinical studies have indicated that the protective effect of mild hypothermia against brain injury may be due to many factors, such as reduced brain metabolism, decreased intracellular acidosis and lactic acid accumulation, inhibition of excitatory amino-acid release and oxidative stress, and the modification of cell death signaling pathways (Polderman, 2008; Gong et al., 2013). In this study, $33^{\circ}-34^{\circ} \mathrm{C}$ was chosen for the induction of mild hypothermia, based on previous studies that demonstrated that mild hypothermia has a potent neuroprotective action (Logue et al., 2007; Park et al., 2013). Lu et al. (2014) demonstrated that re-warming of the body at a slow rate of $0.5^{\circ}-1{ }^{\circ} \mathrm{C} / \mathrm{h}$ preserved 
the beneficial effects of hypothermia on cerebral function. Therefore, this re-warming rate was used in our study.

Cardiac arrest causes an immediate reduction in ATP and phosphocreatine, initiating a switch to glycolytic metabolism. As a result, the intracellular levels of lactate and $\mathrm{H}^{+}$are dramatically increased, leading to both intracellular and extracellular acidosis and $\mathrm{Ca}^{2+}$ influx into the cells. To further exacerbate this injury process, acidosis and the lack of ATP inhibit the cellular mechanisms that are normally responsible for controlling excessive intracellular $\mathrm{Ca}^{2+}$, such as ATP-dependent $\mathrm{Na}^{+} / \mathrm{K}^{+}$pumps and ion channels (Schmitt et al., 2014). However, these harmful processes can be attenuated by hypothermia. In this study, we found that V-ATPase activity decreased after CA. Moreover, V-ATPase activity was increased in the HT- 3 h group compared to the NT-3 h group. These results are consistent with other studies that have demonstrated that hypothermia has a beneficial impact on ion pump dysfunction. Our data further confirmed that mild hypothermia reduces brain injury, even with brief (i.e., $2 \mathrm{~h}$ ) periods of hypothermia.

Acidosis is a common consequence of exposure to CA and hypoxia (Michiels, 2004). It is not yet known how mild and transient acidosis can result in both apoptosis and necrosis. Ding et al. (2000) reported that transient $(15 \mathrm{~min}$ ) acidification of intracellular $\mathrm{pH}$ induces delayed neuronal loss in cultured hippocampal slices; the acidosis-induced necrosis and apoptosis were both blocked by hypothermia. Recent studies have reported that V-ATPase assists in the regulation of cytosolic $\mathrm{pH}$ in neurocytes (Di Giovanni et al., 2010). In neurons, V-ATPase forms a pH gradient across vesicular membranes. Transmitters are taken up through vesicle-associated membrane proteins that are coupled to this gradient (Moriyama et al., 1992). Furthermore, the $\mathrm{pH}$ gradient established by V-ATPase is a driving force for concentrating transmitters inside the organelles. After CPR, the $\mathrm{pH}$ decrease and calcium increase in the cytoplasm can lead to the opening of mitochondrial permeability transition pores and cell death (Crompton, 2000). Thus, V-ATPase may play a key role in the neurocyte loss after CA (Moriyama et al., 1992).

The subunit V0a1 specifically targets presynaptic membranes and is highly expressed in neurons (Morel et al., 2003), which may reflect a specialized role in brain tissue (Su et al., 2003; Poëa-Guyon et al., 2006). A recent report demonstrated that V0a1 plays an important role in regulating neurotransmitter uptake, storage, and release by exocytic vesicles in neurosecretory PC12 cells (Saw et al., 2011). Here, we found that cleaved caspase 3 was up-regulated and V0a1 was reduced in the NT-3 h group after CA compared to the HT-3 h group. The reduced V0a1 subunit expression in the neuronal cells may increase intracellular environment disorders, causing a subsequent cascade reaction, and may explain the delayed necrosis and apoptosis that is induced by cellular acidosis. Our observations are further supported by other reports that demonstrate that concanamycin (a V-ATPase inhibitor) can induce apoptosis in a variety of cells (e.g., tumor cells and osteoclasts) through caspasedependent mechanisms (Aiko et al., 2002; Xu et al., 2003).

There were several limitations to our study. First, V-ATPases are large, multi-subunit, membrane-associated protein complexes. We only examined the V0a1 subunit; therefore, we do not know how the other subunits function under these conditions. Second, we used the transcutaneous electrical epicardium stimulation to establish the CA model in healthy rats. This is in contrast to most cases of CA, where there are concurrent underlying diseases. Third, we did not measure the intracellular $\mathrm{pH}$ in the rat brains, and we were unable to determine the direct effects of V-ATPase down-regulation on the intracellular $\mathrm{pH}$ during the post-ROSC 
period. Therefore, further studies are needed to assure the effectiveness of V-ATPase and determine whether there is a link between activity up-regulation and anti-post-cardiac arrest syndrome. Fourth, our study was designed to investigate an acute-stage change in V-ATPase expression following CA ( $24 \mathrm{~h}$ after ROSC). Further studies are needed to investigate the changes in V-ATPase expression in the later phases after CA.

In this study, we demonstrated both the neuroprotective capacities of mild hypothermia via histological protection and an anti-apoptotic mechanism. Based on the data that suggest that an increase in V-ATPase activity was associated with a decreased expression of cleaved caspase 3, V-ATPase activation may be important for anti-apoptotic protection after CPR. The positive effects of V-ATPase over-expression on neurologic recovery after CPR deserve further investigation.

\section{Conflicts of interest}

The authors declare no conflict of interest.

\section{ACKNOWLEDGMENTS}

Research supported by the National Natural Science Foundation of China (\#81272063).

\section{REFERENCES}

Aiko K, Tsujisawa T, Koseki T, Hashimoto S, et al. (2002). Involvement of cytochrome c and caspases in apoptotic cell death of human submandibular gland ductal cells induced by concanamycin A. Cell. Signal. 14: 717-722. http:// dx.doi.org/10.1016/S0898-6568(02)00016-5

Chintagari NR, Mishra A, Su L, Wang Y, et al. (2010). Vacuolar ATPase regulates surfactant secretion in rat alveolar type II cells by modulating lamellar body calcium. PLoS One 5: e9228. http://dx.doi.org/10.1371/journal.pone.0009228

Chung C, Mader CC, Schmitz JC, Atladottir J, et al. (2011). The vacuolar-ATPase modulates matrix metalloproteinase isoforms in human pancreatic cancer. Lab. Invest. 91: 732-743. http://dx.doi.org/10.1038/labinvest.2011.8

Crider BP and Xie XS (2003). Characterization of the functional coupling of bovine brain vacuolar-type $\mathrm{H}(+)$-translocating ATPase. Effect of divalent cations, phospholipids, and subunit H (SFD). J. Biol. Chem. 278: 44281-44288. http:// dx.doi.org/10.1074/jbc.M307372200

Crompton M (2000). Mitochondrial intermembrane junctional complexes and their role in cell death. J. Physiol. 529: 1121. http://dx.doi.org/10.1111/j.1469-7793.2000.00011.x

Di Giovanni J, Boudkkazi S, Mochida S, Bialowas A, et al. (2010). V-ATPase membrane sector associates with synaptobrevin to modulate neurotransmitter release. Neuron 67: 268-279. http://dx.doi.org/10.1016/j.neuron.2010.06.024

Ding D, Moskowitz SI, Li R, Lee SB, et al. (2000). Acidosis induces necrosis and apoptosis of cultured hippocampal neurons. Exp. Neurol. 162: 1-12. http://dx.doi.org/10.1006/exnr.2000.7226

Fogarty FM, O'Keeffe J, Zhadanov A, Papkovsky D, et al. (2014). HRG-1 enhances cancer cell invasive potential and couples glucose metabolism to cytosolic/extracellular $\mathrm{pH}$ gradient regulation by the vacuolar- $\mathrm{H}(+)$ ATPase. Oncogene 33: 4653-4663. http://dx.doi.org/10.1038/onc.2013.403

Gong P, Hua R, Zhang Y, Zhao H, et al. (2013). Hypothermia-induced neuroprotection is associated with reduced mitochondrial membrane permeability in a swine model of cardiac arrest. J. Cereb. Blood Flow Metab. 33: 928-934. http://dx.doi.org/10.1038/jcbfm.2013.33

Katz L, Ebmeyer U, Safar P, Radovsky A, et al. (1995). Outcome model of asphyxial cardiac arrest in rats. J. Cereb. Blood Flow Metab. 15: 1032-1039. http://dx.doi.org/10.1038/jcbfm.1995.129

Knapp J, Heinzmann A, Schneider A, Padosch SA, et al. (2011). Hypothermia and neuroprotection by sulfide after cardiac arrest and cardiopulmonary resuscitation. Resuscitation 82: 1076-1080. http://dx.doi.org/10.1016/j. resuscitation.2011.03.038

Knapp J, Bergmann G, Bruckner T, Russ N, et al. (2013). Pre- and postconditioning effect of Sevoflurane on myocardial dysfunction after cardiopulmonary resuscitation in rats. Resuscitation 84: 1450-1455. http://dx.doi.org/10.1016/j. 
resuscitation.2013.04.012

Lin JY, Liao XX, Li H, Wei HY, et al. (2010). Model of cardiac arrest in rats by transcutaneous electrical epicardium stimulation. Resuscitation 81: 1197-1204. http://dx.doi.org/10.1016/j.resuscitation.2010.05.019

Logue ES, McMichael MJ and Callaway CW (2007). Comparison of the effects of hypothermia at 33 degrees C or 35 degrees C after cardiac arrest in rats. Acad. Emerg. Med. 14: 293-300. http://dx.doi.org/10.1111/j.1553-2712.2007. $\underline{\text { tb02010.x }}$

Lu X, Ma L, Sun S, Xu J, et al. (2014). The effects of the rate of postresuscitation rewarming following hypothermia on outcomes of cardiopulmonary resuscitation in a rat model. Crit. Care Med. 42: e106-e113. http://dx.doi.org/10.1097/ CCM.0b013e3182a63fff

Marino ML, Fais S, Djavaheri-Mergny M, Villa A, et al. (2010). Proton pump inhibition induces autophagy as a survival mechanism following oxidative stress in human melanoma cells. Cell Death Dis. 1: e87. http://dx.doi.org/10.1038/ cddis. 2010.67

Marshansky V and Futai M (2008). The V-type H+-ATPase in vesicular trafficking: targeting, regulation and function. Curr. Opin. Cell Biol. 20: 415-426. http://dx.doi.org/10.1016/j.ceb.2008.03.015

Michiels C (2004). Physiological and pathological responses to hypoxia. Am. J. Pathol. 164: 1875-1882. http://dx.doi. org/10.1016/S0002-9440(10)63747-9

Morel N, Dedieu JC and Philippe JM (2003). Specific sorting of the a1 isoform of the V-H+ATPase a subunit to nerve terminals where it associates with both synaptic vesicles and the presynaptic plasma membrane. J. Cell Sci. 116: 4751-4762. http://dx.doi.org/10.1242/jcs.00791

Moriyama Y, Maeda M and Futai M (1992). The role of V-ATPase in neuronal and endocrine systems. J. Exp. Biol. 172: 171-178.

Nishi T and Forgac M (2002). The vacuolar (H+)-ATPases--nature's most versatile proton pumps. Nat. Rev. Mol. Cell Biol. 3: 94-103. http://dx.doi.org/10.1038/nrm729

Park KH, Lee KH and Kim H (2013). Effect of hypothermia on coagulatory function and survival in Sprague-Dawley rats exposed to uncontrolled haemorrhagic shock. Injury 44: 91-96. http://dx.doi.org/10.1016/j.injury.2011.11.016

Poëa-Guyon S, Amar M, Fossier P and Morel N (2006). Alternative splicing controls neuronal expression of v-ATPase subunit a1 and sorting to nerve terminals. J. Biol. Chem. 281: 17164-17172. http://dx.doi.org/10.1074/jbc. $\underline{\mathrm{M} 600927200}$

Polderman KH (2008). Induced hypothermia and fever control for prevention and treatment of neurological injuries. Lancet 371: 1955-1969. http://dx.doi.org/10.1016/S0140-6736(08)60837-5

Saw NM, Kang SY, Parsaud L, Han GA, et al. (2011). Vacuolar H(+)-ATPase subunits Voa1 and Voa2 cooperatively regulate secretory vesicle acidification, transmitter uptake, and storage. Mol. Biol. Cell 22: 3394-3409. http://dx.doi. org/10.1091/mbc.E11-02-0155

Schmitt KR, Tong G and Berger F (2014). Mechanisms of hypothermia-induced cell protection in the brain. Mol Cell Pediatr 1: 7. http://dx.doi.org/10.1186/s40348-014-0007-x

Sennoune SR, Bakunts K, Martínez GM, Chua-Tuan JL, et al. (2004). Vacuolar H+-ATPase in human breast cancer cells with distinct metastatic potential: distribution and functional activity. Am. J. Physiol. Cell Physiol. 286: C1443-C1452. http://dx.doi.org/10.1152/ajpcell.00407.2003

Shin L, Basi N, Jeremic A, Lee JS, et al. (2010). Involvement of vH(+)-ATPase in synaptic vesicle swelling. J. Neurosci. Res. 88: 95-101. http://dx.doi.org/10.1002/jnr.22180

Stecker EC, Reinier K, Marijon E, Narayanan K, et al. (2014). Public health burden of sudden cardiac death in the United States. Circ Arrhythm Electrophysiol 7: 212-217. http://dx.doi.org/10.1161/CIRCEP.113.001034

$\mathrm{Su}$ Y, Zhou A, Al-Lamki RS and Karet FE (2003). The a-subunit of the V-type H+-ATPase interacts with phosphofructokinase-1 in humans. J. Biol. Chem. 278: 20013-20018. http://dx.doi.org/10.1074/jbc.M210077200

Xu J, Feng HT, Wang C, Yip KH, et al. (2003). Effects of Bafilomycin A1: an inhibitor of vacuolar H (+)-ATPases on endocytosis and apoptosis in RAW cells and RAW cell-derived osteoclasts. J. Cell. Biochem. 88: 1256-1264. http:// dx.doi.org/10.1002/jcb. 10477 